\title{
Suggestions to Address the Increase in Demand and Costs in General Medicine: Escaping From a "Black Hole" and from Boomerang Effect
}

\author{
Turabian JL* \\ Department of Family and Community Medicine, Health Center Santa Maria de \\ Benquerencia, Spain
}

*Corresponding author: Jose Luis Turabian, Specialist in Family and Community

Medicine, Health Center Santa Maria de Benquerencia, Toledo, Spain, Email: jturabianf@hotmail.com

\section{Review Article \\ Volume 2 Issue 2}

Received Date: April 20, 2019

Published Date: April 26, 2019

DOI: $10.23880 /$ jqhe- 16000120

\section{Abstract}

General practice of care plays a central role in effective and cost-effective health care. General practitioner (GP) has a solid position: each resident is on the list with a GP is accessible to everyone, and GP handles most of the health problems and coordinates the referral to specialized care. But, utilization rates increase, as do their costs, and this may indicate an inefficient use of health services. The typical response is the increase in the supply of resources, which causes a "black hole" or "boomerang" effect. This article exposes the idea that the increase in demand and assistance at the level of general medicine cannot be resolved by increasing the supply of resources, but rather that the organization of medical practice and technical actions are the main causes that increase health demand, which is inefficient, expensive and iatrogenic. It is the existing trends of medicalization that increase the prevalence of the disease and its treatment, when for example, from the beginning treatment it is made with drug associations, with use of higher doses of drugs, with screening and preventive counselling that are not based in scientific evidence, etc. To avoid the increase in demand, and get out of "black hole", the hypothesis is presented that achieving a more reflexive and critical GP, who in every consultation, is prepared to understand and solve the query "in another way", in biopsychosocial terms and avoiding medicalization, and also delegating and working in a team, but keeping leadership of patient management in each clinical problem, can be a real filter for the introduction of medical measures that are little contrasted at the individual and population level, both from the clinical, ethical, quality, epidemiological and economic points of view.

Keywords: Health Care Demand; Health Care Projections; Health Expenditures; Health Services Needs and Demand/Trends; Delivery of Health Care; Physicians/Supply \& Distribution; General Practice

\section{Introduction}

Health depends on some key issues of the existence of health systems, which are a product of society, so that they provide services according to the needs of the population [1,2]. An essential aspect of health systems is primary health care, and within this subsystem is general medicine/family medicine [3]. 


\section{Journal of Quality in Health Care \& Economics}

General practitioners (GPs) are independent professionals, and offer private services with public funding in most developed countries: Germany, Australia, Austria, Canada, Belgium, Denmark, Holland, Ireland, Italy, Japan, New Zealand, Norway, United Kingdom, Singapore, and Switzerland. The usual is the work only practice, with the exception of the United Kingdom, where the work predominates in small groups of 2-3 general practitioners in health centers (of private property, of the general practitioners themselves, in the United Kingdom, Canada, the Netherlands, and in other countries). The GP is a salaried professional and works in publicly owned health centers in Spain, Finland, Portugal and Sweden (and in rural areas of Greece) [4].

In developed countries, about two-thirds of any population visits a family medicine service at least once a year, and more than $80 \%$ contacts once every 5 years [58]. In many of these health systems, the GP is the gatekeeper of health care and coordinator of the patient's trajectory through the system [9].

It is a widely known fact that demand is increasing (number of consultations per doctor) and frequentation (number of consultations per person/year), with increasing increases in health expenditures, and at the same time also the expectations of the population in health systems, considered globally, and especially in family medicine, especially in the places or countries where the GP is the gatekeeper to the system, such as UK, Spain, etc. [10-14].

In this scenario, in relation to the increase in demand and costs, health systems can be equated to "black holes: finite regions of space inside which there is a sufficiently high and dense mass concentration to generate such a gravitational field that no material particle, not even light, can escape from it" [15]. Thus, all Western health systems, for years, are close to collapse "by swallowing increasing amounts of demand and costs," without being able to escape this process.

Thus, analyzes of health systems increasingly focus on the issue of "excessive use" of health services and how to address them. In this article of reflection or opinion, based on the author's own experience and a nonsystematic review or narrative review, we try to conceptualize about this phenomenon of the seemingly unstoppable increase in health demand, and offer approaches to address it from the point of view of general medicine.

\section{Discussion}

Of course, adequate and timely access to high quality care is a cornerstone of primary care [16]. Primary health care is intended to be where people have their "first contact care, health promotion and basic treatment", and their GP is intended to facilitate "adequate access to other medical care and related services for those who need it." However, above a certain threshold of resources or services -above a certain level of supply- we find a "black hole" effect.

\section{The Example of the Curious Paradox of the Lanes on the Highway}

This paradox allows us to reflect on this aspect of unstoppable demand: Since highways are frequently congested, why do not lanes expand on the highway? It is a question with which drivers usually occupy part of the time lost in each traffic jam of an exit operation or with each morning bottling. The answer given by the experts is, perhaps, surprising. Creating wider roads does not relieve traffic jams. In fact, it maintains them and even increases them in the medium term. "Adding highway lanes to deal with traffic congestion is like loosening your belt to cure obesity." With this quote, which is usually attributed to the architect and town planner Lewis Mumford, it is explained $[17,18]$.

If more lanes are created, a phenomenon called "the black hole of the motorway extension" appears. If we doubled the number of lanes (with the consequent high cost), the traffic jam would go away, and people who do not use a car would use it, and finally the traffic jam would return. At the beginning it is better, but the idea of wider motorways "generates a diversion of users from other means of transport, from other routes and other schedules." Those who got up early to avoid rush hour, those who went by bus to work or those who made another car tour stop doing so, and a boomerang effect is generated.

An example of this negative effect is Interstate 405 in California, the busiest highway in the United States. The investment to win in breadth cost 1,900 million dollars (1,680 million euros) in 2016. Despite everything, it remains one of the protagonists of the usual traffic jams that are formed in the city on Thanksgiving and that they become viral each year. Not even his initial results were optimal. If, before the extension, the peak hour of traffic in the afternoon lasted seven hours, it was reduced to five hours. But at critical moments the traffic jams were even bigger with the new lanes already in operation. And the 


\section{Journal of Quality in Health Care \& Economics}

situation is expected to worsen in the near future, as The New York Times pointed out in 2016 [19].

If it is usual that these large investments that we all pay do not generate cost savings, why do they continue to apply? "In the case of motorway lanes, the ideal is to apply deterrent measures that provoke the demand for other forms of mobility, such as free public transport, charge for driving through the center of the cities, or for parking, to prevent people from needing to travel to the city center as they live outside the cities against their will when the work centers continue being in the center, etc. [17].

\section{The Behavior of the Health Supply is Responsible for the Demand}

Usually what is said is that the epidemic of obesity, the increase of chronic diseases and aging and the continuous growth of the population, etc. they will combine to create a much greater demand for medical services at the same time that the relative size of the physician's workforce will begin to decrease. Given the urgency of the situation, some authors are calling for an increase in the number of entry-level positions in the physician training system, to produce the necessary doctors to meet the growing demand for medical care. It is said that otherwise, "the perfect storm" will soon disrupt the health care systems [20]. It's like asking for more lanes for the congested highway.

However, there is a large amount of data that shows that the behavior of the health supply is responsible for the demand: the workload for all GPs increases when new doctors are incorporated into the area; an increase of 5$10 \%$ in the number of doctors increases the volume of work by approximately $4 \%$, thus not reducing or doing it so very little, the volume of work per GP, and with large increases in the overall cost. There is especially an increase in second visits within the same episode of illness [5].
In the health claim is really the GP who makes the choice of the quantity demanded, not the patient/ consumer: the latter is limited to seek medical advice when there is an episode of disease that he considers abnormal for his age, cultural norms, sex, social class, etc., but having no information or capacity to restore their conditions, their choice is limited to accepting or not the indications of the GP who is the one who chooses the amount and type of assistance [5].

In addition, in this sense, there is abundant data indicating the more people are exposed to contemporary health care, the sicker they feel. GPs do not understand the effects of being labeled at risk. And on the other hand, more money can be made from selling healthcare interventions for the healthy majority than for the sick minority [21].

Therefore, if the increase in services and doctors is counterproductive, what alternative measures can be useful to avoid increasing demand?

\section{Suggestions for Addressing the Increase in Demand in General Medicine}

How to control the demand and/or excessive frequentation? General practice care plays a key role in keeping healthcare effective and cost-efficient. However, variation in the utilization rates of practices may reveal variation in practice performance. Utilization rates of general practice care can be partially explained by the socio-demographic profile of the patients' area of residence, but also and mainly by practice organization characteristics. Responding to the demand (responding to the patient's query) bio medically leads to a greater increase in demand (new queries repeated for the same reason and new consultations for other reasons) [5,22-27] (Figure 1). Table 1 presents the possible tools to control the demand in general medicine. Table 2 presents a more specific and concrete list of clinical and organizational tasks.

\section{Methods And Tools To "Control Demand" In General Medicine}

\begin{tabular}{|c|l|}
\hline 1 & Be properly placed on the field of play \\
\hline 2 & Prepare the consultation \\
\hline 3 & $\begin{array}{l}\text { It is not a question of arriving at biomedical diagnoses that can be classified in the ICD 10, but to arrive at } \\
\text { "understanding from the bio-psycho-social point of view" the patient }\end{array}$ \\
\hline 4 & Delegate and work as a team, keeping the family doctor as the professional in charge of patient management \\
\hline 5 & Resolving the demand "otherwise non-medicalizing" \\
\hline
\end{tabular}

Table 1: Methods and Tools to "Control Demand" in General Medicine. 


\section{Journal of Quality in Health Care \& Economics}

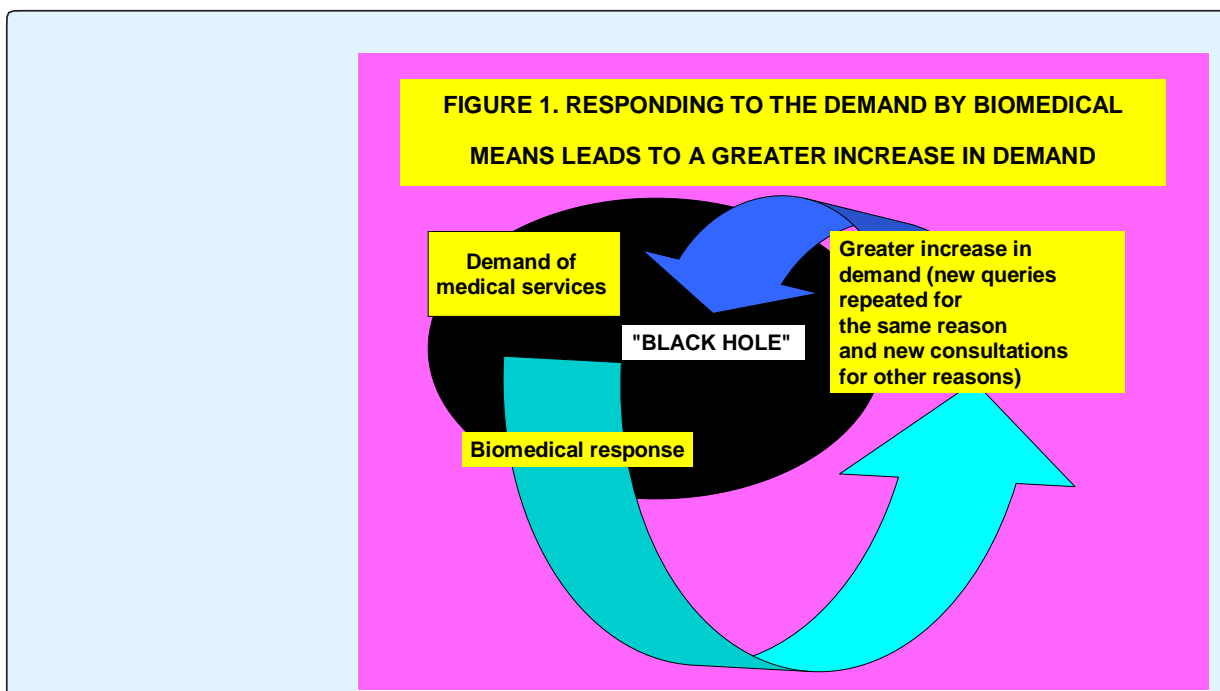

Figure 1: Responding To the Demand by Biomedical Means Leads to a Greater Increase in Demand.

\section{Specific and Concrete List of Clinical and Organizational Tasks to Control the Demand in General Medicine}

$1 \quad$ Think about whether a complementary test is required or not before requesting it; If you think that test will not change a decision, do not ask for it

2 Do not say to the patient "come back in three days" if it is not necessary: you can be creating a dependency of the patient towards the useless and medically dangerous doctor

3 Do not prescribe much. Over-prescribing produces polypharmacy, adverse drug reactions, iatrogenesis ... and more consultations of your patients that you are creating yourself

$4 \quad$ Do not use new drugs as a rule; wait for them to consolidate. Family medicine is not the place to experiment with new drugs

5 Do not prescribe drugs if it is not precise or its indication is not clear; Do not start a treatment with two or more drugs if you can use only one; Do not use high doses of drugs without clear reasons

\begin{tabular}{|l|l|}
\hline 6 & $\begin{array}{l}\text { Program and schedule all the visits you can: chronic problems, collection of tests, reviews, recipes, etc. Do not } \\
\text { leave the date of new visits of your patients open for them to choose; They generally tend to bring visitors } \\
\text { closer together and increase attendance. When scheduling an appointment, the patient tends to comply with it } \\
\text { and to join several consultations in that one visit }\end{array}$ \\
\hline 7 & $\begin{array}{l}\text { Refer the patient to the nurse, to the social worker, to the hospital specialists, as appropriate. Do not believe } \\
\text { that you are alone or that you "must" solve alone everything; It is counterproductive: the experts in each area } \\
\text { solve it better and you will not overload yourself }\end{array}$ \\
\hline 8 & $\begin{array}{l}\text { Be integral in each visit; do routine anticipatory care: do genograms, prepare the visit, review all problems } \\
\text { (current, chronic, preventive, recipes, etc.), even if the patient comes only for one topic }\end{array}$ \\
\hline 9 & $\begin{array}{l}\text { Do not visit a chronically well-controlled patient very frequently. Does controlled hypertension need to be seen } \\
\text { every month or every 4 months or every 6 or every year? }\end{array}$ \\
\hline 10 & Do not ask for additional tests "just in case". Remember the concepts of sensitivity and specificity \\
\hline 11 & Do not forget that in general medicine, you must first go to the "context" and then to the complaint \\
\hline 12 & $\begin{array}{l}\text { Do not forget that in general medicine you should go to the biological only when you have discarded the } \\
\text { psychosocial (it is the opposite of the usual in the hospital!) }\end{array}$ \\
\hline
\end{tabular}

Table 2: Specific and Concrete List of Clinical and Organizational Tasks to Control the Demand in General Medicine. 


\section{Journal of Quality in Health Care \& Economics}

Be properly placed on the field of play: The attention of the demand of each day (especially in the national health services where the visit of the patient to the GP is free) can exhaust the doctor.

We can compare the care or resolution of this demand with the game of tennis or soccer game; a good placement and concentration of the player is necessary to win the game. If every ball that does arrive (in a game of tennis or football) catches us dislocated, we can only try, with great effort, to get the ball and try to return it or take the ball away. Maybe we can return one ball, but the next one takes us wrong placed, and we still have to make a bigger effort to return it. And so on. This situation puts us continually on the verge of losing the game and it greatly fatigues us (the doctor's burnout); we can no longer enjoy the game, just try to survive at the cost of great physical effort. After each ball returned in extremis, we are even worse placed in the field.

This is what happens in the GP consultation that has not been prepared in some way the query of each patient (review who is that patient and where is and what has until then, what are until then their problems "with energy"? What treatment plans have been made? What treatments, what previous results of tests and visits to specialists, etc?.

Before each patient goes to the consultation, GP has to concentrate and "stand" in a position to properly "receive and respond" to the patient.

If GP recive the patient each time he comes to the office, as a "new" visit (right from "zero"; without taking into account the previous continuous care) and meet the demand as a punctual visit, without any previous preparation, having to make very quick decisions, in a matter of minutes, in the visits of a duration of between 610 minutes that occur in a large part of the European countries in general medicine, and with the great demand of 30 or 50 visits every day, puts us on the edge, at each visit, to respond to the problem posed by the patient in a way precipitate or incorrect ("dislocated on the field").

This means that we prescribe drugs or ask for unnecessary or not indicated complementary tests, which lead us to be even more "unsettled" in the next visit of that patient, since the adverse effects, the false positive findings, etc. appear, and we also go generating more demand, with which we close the vicious circle of being worse and worse placed to be able to respond adequately to the demands of each patient, and we "burn", and finally make mistakes in diagnosis and treatment ("we lose the game").

Prepare the consultation before entering the patient, memorizing the patient's previous history: We must have in our mind the patient's previous biopsychosocial history when he or she entering the consultation, so that we do not need to read computer screens, not written documents, etc. In this way, the doctor can focus all his attention on the patient and can integrate his verbal and non-verbal communication, mentally in his previous clinical history, immediately, which will allow us to be more effective and will be understood by the patient as we recognize and remember him as a person and as a sick person (affective and cognitive competence of the doctor).

Before each patient goes into to the consultation, the GP has to concentrate and "position" himself in a suitable position to adequately "receive and respond" to the patient. So, check the personal history or list of the patient's problems, their last visits with the GP (based on the continuity of care), and visits with other specialists, their treatments, their work and family situation, etc. After this, GP "almost" can predict the reason for visiting to doctor in a large part of the patients $[5,28]$.

It is not about reaching biomedical diagnosis that can be classified in the ICD 10, but reaching "understanding from the biopsychosocial point of view" the patient: Some practical tools of the biopsychosocial model to be applied in the tasks of consulting each day in family medicine are:

- Interview (from the context to the symptom, decentralized and contextualized interview).

- Contextualized Clinical History (list of problems, resources, and beliefs, list of "problems-force", grids of evaluation biopsychosocial of the patient, data collection of several actors, use of qualitative instruments in the taking of the clinical history).

- Contextualized decisions (the value of continuity of care, experience, emotions, patient participation, etc.).

- Other classical instruments of family medicine (family life cycle, genogram, stressful events / family crises; turning points and transitions).

- Other classic instruments of community medicine (scales of social support, risk / morbidity and mortality groups), inventories of community resources (mutual help groups, etc.), relational capital, inventory of patients-resources, and group consultations [29-33]. 


\section{Journal of Quality in Health Care \& Economics}

But, if the GP spends time preparing the consultation, and more time to make it biopsychosocial, will it be delayed and there will be a waiting list and more demand pressure? Experience indicates that this is not the case. Once the consultation is organized in this way, when the patient's problems are fully understood and resolved, the demand decreases (there are fewer repeat visits for reasons already attended, and fewer new visits) [5].

Delegate and work as a team, keeping the family doctor as the professional in charge of patient management: Work in medicine is a shared one, literally a collaboration work with all parties involved in health. Collaborate or cooperate, it means coming to support others in their activities. In nature, the formula for survival lies not in power but in symbiosis. It is a process where different parties who see different aspects of a problem can constructively explore their differences and reach solutions that go beyond their own limited vision [34].

Many times the treatment of patient is done by a large "team" of doctors, but often they do not talk to each other, nor show any interest in doing so [35]. Guaranteeing the continuity of care is essential for proper treatment of patients. The patient can be seen in the acute care units or in the outpatient clinics of the hospital, but most of the time it is under the supervision of GP. In addition, several specialists in the management of the patient often participate, and patient can change their domicile when they are rotated with different family members (especially the elderly patient), and therefore with different primary care team.

Patients can be treated by many different doctors, even for the same pathology. If there is no good coordination, diagnostic and treatment errors, as well as failure to comply with the treatment is guaranteed. An adequate clinical report can help. But it is essential to establish adequate channels of communication between the different doctors and the patient, which are quick and comfortable. A fluid communication avoids therapeutic breaches, adverse reactions to medications, visits to the emergency room, hospital admissions etc. [36].

Resolve the demand "otherwise non-medicalizing": It means not medicalizing; avoid biomedical responses for each query. It means incorporating the nuance of contextualization in each query; Take into account actors, relationships, resources and contexts, and use this knowledge for therapeutic intervention; Increase patient's ability to cope with the health problem, strengthen self-responsible compliance, skills to deal with emotional problems, favoring the patient's rights with respect to the social system (work, learning, living conditions ...) [37].

This process towards contextualization in the medical response includes: 1 . Going from sickness assistance to health promotion; 2 . Go from the patient's demand to a comprehensive health project; and 3. Go from the biological to the biopsychosocial.

It implies a new type of GP-patient relationship: not seeing a single actor without resources, but different actors with relationships and resources; not to see them as passive receivers of a service, on which they act, but active collaborators in the services; The GP must go from being a "dispenser" of services to a "collaborator" in services. The GP must avoid negative factors and focusing on resources, strengths and positive factors of the patient, changing attitudes, negotiating, providing alternatives, from the immediate concrete assistance objectives to long-term objectives, giving responsibility to the patient, learning, and accepting. The GP should avoid immediate biomedical responses and satisfactions, creatively using the difficulty, responding in an integral way.

\section{Resolving the Demand for "Another Way" also implies:}

Try to see each clinical problem in terms of the cultural reality of the disease, where the patient's perception and motivation are taken into account as intrinsic to their clinical condition, as a microcosm of their culture, and the treatment plan is elaborated in harmony with these individual and community feelings.

$>$ Begin to think in terms of groups: looking for group responses to individual demands.

$>$ Provide the person who requests our help, from the recognition of their difference, instruments to control their own situation.

$>$ To understand that the individual and family clinical history should be a rich source of material about the culture and the relationships between health and illness, as well as the vital experiences in that culture and those individuals.

$>$ From a fragmented intervention to an integral one

$>$ From a distant position to another implied

$>$ Related to patients in terms of network

$>$ Developing an new model of coexistence away from the defensive attitude of the warrior and open to decentralization and diversity [37]. 


\section{Journal of Quality in Health Care \& Economics}

\section{Conclusion}

General practice plays a central role in effective and cost-effective health care. When utilization rates increase, as do their costs, this may indicate an inefficient use of health services. The typical response is the increase in the supply of resources, which causes a "black hole" or "boomerang" effect. But, it is the practice organization characteristics and the medical technical actions that are the main cause of the ineffective, expensive and iatrogenic health demand increase. The hypothesis is presented (Figure 2) that achieving a more reflexive and critical GP can be a true filter to the introduction of medical measures little contrasted at the individual level and clearly pathological at the population level, from the point of view of quality, epidemiology, and economy. It is about the GP, in every consultation, being prepared to understand and solve the query "in another way", in biopsychosocial terms and avoiding medicalization, in the cultural reality of the disease, where perception and motivation of the patient are considered an element intrinsic to clinical condition, and the treatment plan is elaborated in harmony with these individual and community feelings, and also know how to delegate and working in a team, but keeping leadership of patient management in each clinical problem.

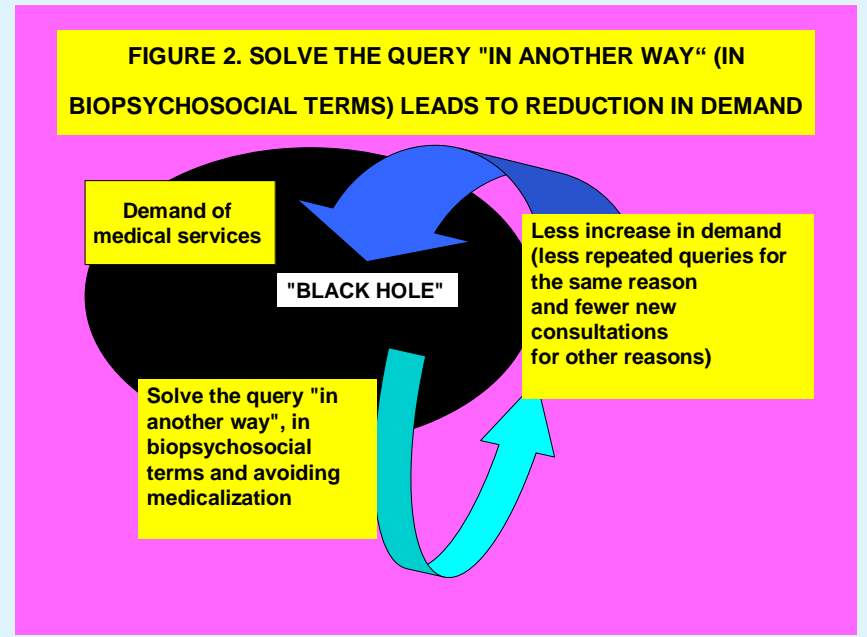

Figure 2: Solve the Query "in Another Way"(In Biopsychosocial Terms) Leads to Reduction in Demand.

\section{References}

1. Gérvas J, Pérez Fernández M (2015) Non-clinical miscellany: health systems are part of social determinants. From Canada to Greece, passing through the United States. AMF 11(4): 233-240.

2. Gérvas J (2012) Primary health care oriented systems, for whom, why and how. In: A symposium celebrating the scientific and policy legacy of Bárbara Starfield. Johns Hopkins Bloomberg School of Public Health, Baltimore.

3. Hone T, Macinko J, Millett C (2018) Revisiting AlmaAta: what is the role of primary health care in achieving the Sustainable Development Goals? Lancet 392(10156): 1461-1462.
4. Gérvas J, Pérez Fernández M (2006) Strong Primary Care: clinical, epidemiological and social basis in developed and developing countries. Rev bras epidemiol 9(3).

5. Turabian JL (1995) Family and community medicine notebooks: an introduction to the principles of family medicine. Ediciones Díaz De Santos 1: 576.

6. Hart JT (1981) A new kind of doctor. J R Soc Med 74(12): 871-883.

7. Hart JT (1988) A new kind of doctor. The General Practitioner's part in the health of the community (review) 33(2): 310-311.

8. Hippisley Cox J, Vinogradova Y (2009) Trend in consultation rates in general practice 1995 to 2008 : 


\section{Journal of Quality in Health Care \& Economics}

analysis of the QRESEARCH database: the NHS Information Centre, pp: 1-23.

9. Boshuizen HC, Poos MJJC, van den Akker M, van Boven K, Korevaar JC, et al. (2017) Estimating incidence and prevalence rates of chronic diseases using disease modeling. Popul Health Metr 15: 13.

10. Blair A (2004) Meeting increased demand. Jpn Hosp 23: $19-22$.

11. Choi Y, Fabic MS, Hounton S, Koroma D (2015) Meeting demand for family planning within a generation: prospects and implications at country level. Glob Health Action 8: 29734.

12. Economou C, Kaitelidou D, Karanikolos M, Maresso A (2017) Greece: Health System Review. Health Syst Transit 19(5): 1-166.

13. Gaal P, Szigeti S, Csere M, Gaskins M, Panteli D (2011) Hungary health system review. Health Syst Transit 13(5): 1-266.

14. T Sánchez Sagrado (2017) Primary care in Belgium. Semergen 43(6): e57-e60.

15. Hawking SW, Ellis GFR (1973) The Large Scale Structure of Space-time, Cambridge, Cambridge University Press.

16. Starfield B, Shi L, Macinko J (2005) Contribution of primary care to health systems and health. Milbank Quart 83(3): 457-502.

17. Llanos Martínez H (2019) The motorways of more lanes do not avoid traffic jams.

18. (2010) Talk: Lewis Mumford.

19. Nagourney A (2016) Los Angeles Drivers on the 405 Ask: Was $\$ 1.6$ Billion Worth It?.

20. Whitcomb ME (2011) Commentary: Meeting future medical care needs: a perfect storm on the horizon. Acad Med 86(12): 1490-1491.

21. Heath I (2005) Who needs health care the well or the sick? BMJ 330: 954.

22. de Graaf-Ruizendaal WA, van der Hoek L, de Bakker DH (2018) Are low and high utilization related to the way GPs manage their practices? An observational study. BMC Family Practice 19: 46.
23. Moriah E Ellen, Michael G Wilson, Marcela Vélez, Ruth Shach, John N Lavis, et al. (2018) Addressing overuse of health services in health systems: a critical interpretive synthesis. Health Res Policy Syst 16: 48.

24. McCarthy M (2018) Consistently high consultation rates every day are not safe for doctor or patient. BMJ 361: k2545.

25. Yeung EYH, Mohammed RSD (2018) Learning from the Canadian experience of capping GP consultation rates. BMJ 361: k2550.

26. Webster M, Stewart R, Aagaard N, McArthur C (2019) The learning health system: trial design and participant consent in comparative effectiveness research. Eur Heart J 40(15): 1236-1240.

27. Turabián JL, Perez Franco B (2014) Reflections on the present and future of family medicine. Gac Sanit 28(3): e5-e8 (181-262).

28. Turabian JL (2018) Twelve Commandments for General Medicine Clinical Record: Some Suggestions That Are Not in the Text books. Archives of Community and Family Medicine 1(2): 21-29.

29. Turabián JL, Pérez Franco B (2008) Individual health care with community orientation - contextualized care: The figure is the background. Revista Clínica Electrónica en Atención Primaria, pp: 1-5.

30. Nakao M (2017) Somatic manifestation of distress: Clinical medicine, psychological, and public health perspectives. Biopsychosoc Med 11: 33.

31. Turabian JL (2017) Psychosocial factors and organic disease: Two sides of the same coin. Chronicle of Medicine and Surgery 1(1): 44-46.

32. Engel G (1980) The clinical appplication of the biopsychosocial model. Am J Psychiatry 137(5): 535544.

33. Turabián JL, Pérez Franco B (2005) A way to make clinical pragmatism operative: Sistematization of the actuation of competent physicians. Med Clin (Barc) 124(12): 476.

34. Turabian JL (2016) Teamwork Between Gynecology and Family Medicine: The Tennis Players in a Doubles Match and the Fable of the Butterfly and the Beetle. Gynecol Obstet 6: e119. 


\section{Journal of Quality in Health Care \& Economics}

35. Pujols McKee A (2013) Taking Care of My Parents. JAMA Intern Med 174(3): 323.

36. Adelman RD, Greene MG, Ory MG (2000) Communication between older patients and their physicians. Clin Geriatr Med 16(1): 1-24.
37. Turabian JL, Perez Franco B (2001) Community Activities in Family Medicine and Primary Care. 